\title{
Mutter-Vater-Kind-Kuren der GKV
}

\section{BERNARD BRAUN JÖRN SOMMER, STEFAN MEYER}

Dr. Bernard Braun ist assoziierter Gesundheitswissenschaftler am SOCIUM Forschungszentrum Ungleichheit und Sozialpolitik der Universität Bremen

Dr. Jörn Sommer ist Sozialwissenschaftler und Bereichsleiter der InterVal GmbH, Berlin

Stefan Meyer ist Politikwissenschaftler und Wissenschaftlicher Mitarbeiter der InterVal GmbH, Berlin

18,9 Prozent aller Mütter, Väter und pflegenden

Angehörigen benötigten bereits vor der Pandemie eine Rehabilitations- oder Vorsorgemaßnahme. Zahlreiche Studien zeigen, dass sich dieser Bedarf - besonders der der Mütter - mit großer Wahrscheinlichkeit durch die Pandemie erheblich erhöhen wird. In Befragungen bewerten Ärzt:innen wie ehemalige Patient:innen die Wirkung der von der GKV angebotenen spezifischen Mütter-VäterKind-Maßnahmen zumindest kurz- bis mittelfristig positiv, weisen aber auch auf ihre nicht ausreichende Nachhaltigkeit und zahlreiche Defizite im Bereich der Nachsorge hin. Um dem Bedarf „nach der Pandemie“ gerecht werden zu können, sind Reformen beim Leistungsangebot, der Organisation und Finanzierung von Nachsorge notwendig.

\section{Einleitung}

$\mathrm{Zu}$ den durch die Corona-Pandemie und die politisch verordneten Maßnahmen gesundheitlich wie sozial besonders betroffenen Personengruppen gehören Kinder und ihre Eltern (Sommer et al. 2021: S. 164-181; OECD 2021; Bujard et al. 2021; RKI 2020, Bastin/Unzicker 2021) und aus diesen Gruppen besonders alleinerziehende Eltern, pflegenden Familienangehörige oder Familien aus unteren sozialen Schichten und die Mütter.

Ein Teil der innerfamiliären Arrangements der schon davor nicht unbelasteten Angehörigen dieser sozialen Gruppen (besonders erwerbstätige Mütter) erwiesen sich im Kontext der Pandemiebewältigung innerhalb weniger Wochen als brüchig. Dies vor allem deshalb, weil durch den stetigen Ausbau der Schulpflicht hin zur Ganztagsschule und das gesetzliche Recht auf Kindertagesstättenbetreuung ein Teil der trotz vieler Gleichberechtigungs-Rhetorik Müttern überlassenen familiären Betreuungsarbeit verlässlich externalisiert erschien. Die notwendige Übernahme der Betreuung von Kindern und die durch Kurzarbeit etc. verschlechterte finanzielle Situation von Eltern verschlechterte den körperlichen, psychischen oder mentalen Gesundheitszustand von Müttern erheblich (siehe dazu u.a. Bujard et al. 2021).

Dabei handelt es sich nicht nur um die negativen Folgen der Pandemie und damit um ein mit ihrem absehbaren Ende oder ihrer Rückentwicklung verschwindendes soziales Phänomen, sondern in hohem Maße um die negativen Folgen von geschlechtsspezifischen Unterschieden bei innerfamiliären Normen und Lastenverteilung (OECD 2021: S.2).

Welche Grundlast aus familienspezifischen sozialen Belastungen und dadurch geförderten gesundheitlichen Beeinträchtigungen bereits vor der Pandemie in Familien und darin besonders für Mütter aber auch für Väter, Kinder und nicht zuletzt für pflegende Angehörige existierte und wie die spezifische gesundheitsbezogene Versorgung aussieht, soll im Folgenden näher beleuchtet werden. Diese Grundlast wird möglicherweise nach Beendigung der Pandemie die Lebensverhältnisse dieser Gruppen wieder bestimmen. Als Grundlage dienen mehrere standardisierte Befragungen, die für die vom Bundesministerium für Familie, Senioren, Frauen und Jugend geförderte „Studie zur Untersuchung der Bedarfe von Müttern/Vätern und pflegenden Frauen 
und Männern (mit und ohne Kinder im Haushalt) in Vorsorge- und Reha-Maßnahmen in Einrichtungen des Müttergenesungswerkes" (Sommer et al. 2021) in den Jahren 2019 und 2020 durchgeführt wurden. Dazu zählen Befragungen von 3.799 (explorativ) und 1.330 (intensiv) Müttern, Väter, pflegenden Angehörigen, 139 niedergelassenen Ärzten/ Ärztinnen, 671 ehemaligen Patientinnen/Patienten, die zwischen Oktober 2019 und Februar 2020 eine stationäre Rehabilitations- und Vorsorgemaßnahme in klinischen Einrichtungen des Müttergenesungswerks (MGW) in Anspruch genommen hatten, $48 \mathrm{MGW}$ Einrichtungen zwischen Mai und September 2020 und 352 von 960 Beratungsstellen verschiedener Wohlfahrtsverbände (vgl. Sommer et al. 2021: S. 17ff.).

\section{Normative Grundlagen für spezifische Versorgungsleistungen}

Lang und kurvenreich war der Weg von einer nahezu exklusiven normativen Hervorhebung von Familie (und darin noch zusätzlich der Mütter) im Artikel 6 Abs. 1 und 4 des Grundgesetzes von 1949 bis zum Anspruchsrecht und zur Pflicht der Gesetzlichen Krankenversicherung (GKV)

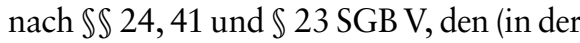
Regel nicht erwerbstätigen) Müttern und Vätern sowie ihren sie bis zum zwölften Lebensjahr begleitenden oder ihren rehabedürftigen Kindern und pflegenden Angehörigen stationäre Rehabilitations- und Vorsorgeleistungen anbieten zu müssen.

Charakteristisch ist bereits, dass unmittelbar nach Inkrafttreten des Grundgesetzes nicht staatliche Einrichtungen oder Sozialversicherungsträger diesen besonderen Schutz und die Fürsorge für Mütter mit Leben bzw. Leistungen füllte, sondern die 1950 gegründete, einzigartige EllyHeuss-Knapp-Stiftung/Deutsches Müttergenesungswerk, deren Einrichtungen bis zum heutigen Tag einen wesentlichen Anteil der Leistungen für Mütter und die hinzugekommenen Zielgruppen der Väter und pflegenden Angehörigen erbringen.

In der seit 1911 und in mehrfach veränderter Weise auch in der alten Bundesrepublik bis 1989 geltenden Reichsversicherungsordnung $(\mathrm{RVO})$ finden sich keine
Pflichtleistungen für Mütter oder gar Väter, sondern Kurleistungen für Genesende, zu denen auch Mütter gehören konnten. Bei der Leistung und ihrer Finanzierung handelte es sich laut der $\$ \mathbb{S} 184$ a und 187 RVO um Satzungs- und Kannleistungen.

Seit 1955 förderte die Bundesregierung die Arbeit des MGW laut einer Antwort auf eine parlamentarische Anfrage im Jahr 1978 mit bis dahin rund 66 Millionen DM aus dem Bundeshaushalt sowie seit 1974 mit 13,5 Mio. DM aus diversen Sonderprogrammen (Deutscher Bundestag. Drucksache 8/1962). Diese Mittel waren „Zweckbestimmt für die Ausstattung und Rationalisierung der Heime einschließlich Baumaßnahmen“ (ebd.) - eine Beschränkung, die bis heute die Förderpraxis des Bundesfamilienministeriums bestimmt.

Laut $\mathbb{} 1$ des Ersten Buch des Sozialgesetzes (SGB I) von 1975 sollen die Sozialversicherungsträger u.a. „dazu beitragen (...) die Familie zu schützen und zu fördern“ und „dazu beitragen, dass (...) die erforderlichen sozialen Dienste und Einrichtungen rechtzeitig und ausreichend zur Verfügung stehen.“

Das Gesetz zur Strukturreform im Gesundheitswesen von 1988 bestimmt Rehabilitationsleistungen für Mütter unter der bereits in der Reichsversicherungsordnung (RVO) geltenden Überschrift „Müttergenesungskuren“ als Kann- oder Ermessensvorschrift und ihre Bezuschussung als Satzungsleistung.

Bereits im Gesetz zur Reform der gesetzlichen Krankenversicherung ab dem Jahr 2000 wird die Überschrift des $\mathbb{4} 4$ SGB V in „Medizinische Rehabilitation für Mütter" umbenannt und auch im Pa- ragrafen wird auf den Rehabilitationscharakter der „stationäre Vorsorge- oder Rehabilitationsmaßnahme" hingewiesen.

Im Gesetz zur Verbesserung der Vorsorge und Rehabilitation für Mütter und Väter von 2002 wurden Väter in den $\mathbb{S} \mathbb{S} 24$ und 41 SGB V ausdrücklich als Leistungsberechtigte für Rehabilitationsoder Vorsorgemaßnahme genannt.

Auch wenn bereits zuvor Mütter und Väter die speziellen Rehabilitations- und Vorsorgeleistungen als Kann-Leistungen beantragen konnten und überwiegend bewilligt bekamen, bestimmte erst 2007 das GKV-Wettbewerbsstärkungsgesetz, einen Anspruch von Müttern und Vätern auf Leistungen nach $\mathbb{} 41$ SGB V als Pflichtleistung. Im selben Gesetz wurde für diese Leistungen der bei anderen GKV-Leistungen geltende Vorrang von ambulanten vor stationären Leistungen aufgehoben.

Erst mit dem Pflege-NeuausrichtungsGesetz von 2012 erhielten pflegende Angehörige das Recht auf dieselben Leistungen wie Mütter und Väter.

\section{Eckdaten zu den Rehabilitations- und Vorsorgeleistungen für Mütter und Väter 2007 bis 2019}

Die Anzahl der stationären Rehabilitations- und Vorsorgefälle ${ }^{1}$ von Müttern und Vätern in der GKV entwickelt sich zwischen 2007 und 2019 unterschied-

1 Auch wenn der Schluss von Fällen auf Personen nur vorsichtig erfolgen sollte, ist der im Falle dieser Leistungsarten mit einem in der Regel mehrjährigen Regelintervall zulässig. Dass eine Person in einem Jahr mehrfach eine dieser Leistungen in Anspruch nimmt, ist unwahrscheinlich. 


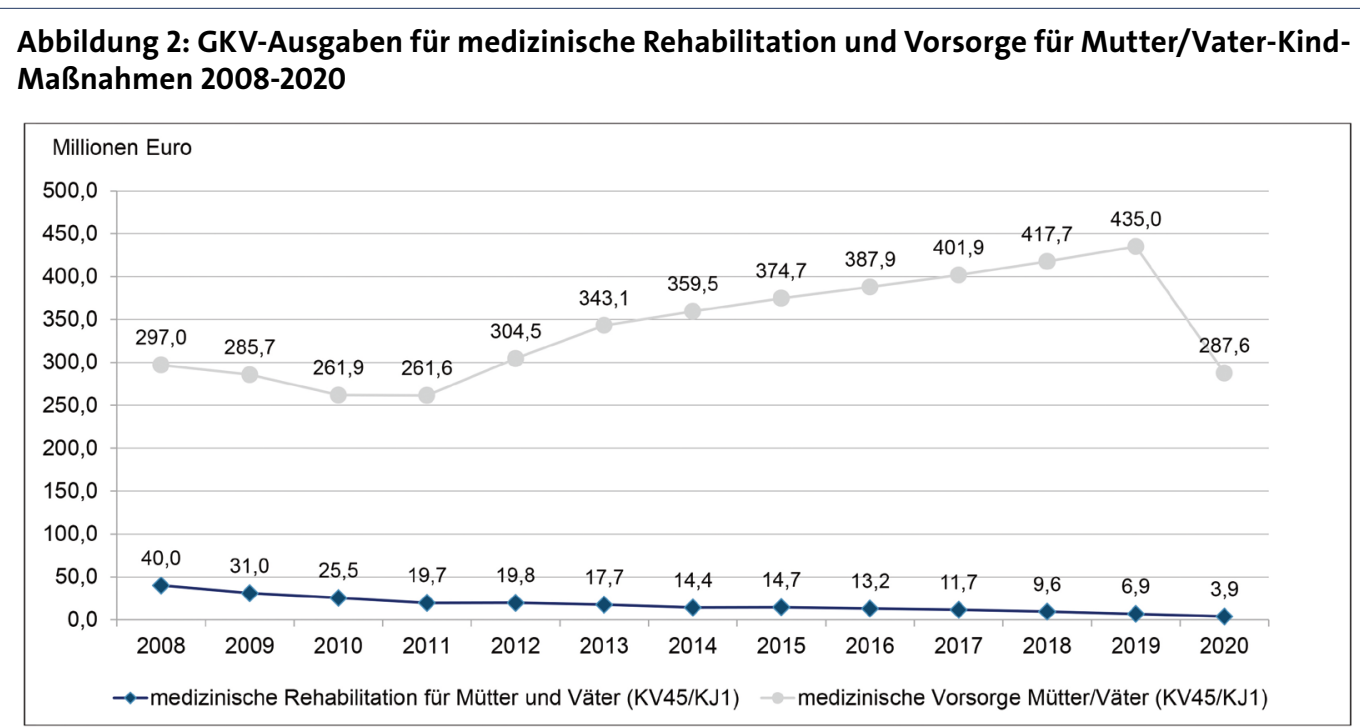

Quelle: Eigene Berechnungen auf Basis KJ1-Statistik, Sommer et al. (2021): S.23 en, wurden Befragungsergebnisse mit objektiven ${ }^{3}$ klinischen Daten oder in anderen repräsentativen Bevölkerungsgruppen erhobenen Daten verglichen. Für die Angaben zur subjektiven Gesundheit und zur Häufigkeit oder Behandlungsprävalenz der von uns abgefragten Erkrankungen waren solche Vergleiche meist möglich. Sie bestätigen überwiegend die Ergebnisse unserer Befragung. Für die Gesundheitsstörungen waren Vergleiche mit unabhängigen Quellen wegen deren starken Situationsabhängigkeit schwieriger, lich. Auf einem bereits sehr niedrigen Niveau geht die Anzahl der RehabilitationsPatient:innen stetig zurück. Die Anzahl von Vorsorge-Patient:innen nimmt dagegen mit einigem zwischenzeitlichem Auf und $\mathrm{Ab}$ im selben Zeitraum insgesamt zu. zahlen schlägt sich im Niveau und Volumen der Ausgaben für diese Leistungen nieder. Zwischen 2008 und 2020 sinken die Ausgaben der GKV für Mutter-/Vater-KindDagegen steigen die Ausgaben für Vorsorgemaßnahmen nach einem Rückgang bis zum Jahr 2011 auf einen Höchstwert im Jahr 2019, um dann wohl pandemiebedingt im Jahr 2020 kräftig abzunehmen.

Die Rehabilitation- und Vorsorgeleistungen für Mütter, Väter und Kinder wurden 2019/20 in insgesamt 132 Einrichtungen erbracht, von denen 73 Einrichtungen im MGW sind (https:// www.kurklinikverzeichnis.de/).

Tabelle 1: Eckdaten zu Eltern-Kind-Maßnahmen für Rehabilitation und Vorsorge

\begin{tabular}{|l|c|c|c|c|}
\hline Jahr & $\begin{array}{c}\text { Ablehnungsquote der } \\
\text { Erstanträge für } \\
\text { MGW-Maßnahmen (in \%) }\end{array}$ & $\begin{array}{c}\text { Anzahl Mütter } \\
\text { in MGW- } \\
\text { Maßnahmen }\end{array}$ & $\begin{array}{c}\text { Anzahl Väter } \\
\text { in MGW- } \\
\text { Maßnahmen }\end{array}$ & $\begin{array}{c}\text { Anzahl Kinder } \\
\text { in MGW- } \\
\text { Maßnahmen }\end{array}$ \\
\hline 2002 & 32 & 50.000 & & 68.000 \\
\hline 2007 & 28 & 43.000 & 900 & 63.000 \\
\hline 2011 & 35 & 39.000 & 1.000 & 56.000 \\
\hline 2012 & 19 & 44.000 & 1.500 & 64.000 \\
\hline 2015 & 11 & 49.000 & 1.600 & 72.000 \\
\hline 2018 & 11 & 48.000 & 2.100 & 71.000 \\
\hline 2019 & 11 (Mütter) / 17 (Väter) & 47.000 & & 70.000 \\
\hline
\end{tabular}

Quelle: MGW (2020)
Die Entwicklung der Patient:innenRehabilitation auf ein geringes Niveau.
Die nur für die Einrichtungen des MGW veröffentlichten differenzierten Daten zeigen, dass die Ablehnungsquote von Erstanträgen seit 2002 um zwei Drittel abnahm, die Anzahl der Patientinnen und Patienten insgesamt und der Kinder in etwa gleich blieb sowie seit 2011 die der Väter stetig zunahm.

\section{Bedarfe}

Unsere Untersuchung zum Bedarf stationärer Vorsorge- oder Rehabilitationsleistungen in Mütter-/Väter/-KindEinrichtungen basiert auf der Erfragung der selbst wahrgenommenen subjektiven Gesundheit und der selbst berichteten Gesundheitsstörungen, Erkrankungen und Belastungen von Müttern, Vätern und pflegenden Angehörigen. Um der möglichen Kritik zu begegnen, dass die damit gewonnenen Aussagen über den Gesundheitszustand per se verzerrt seiin der Tendenz plausibilisieren auch hier externe Daten unsere Ergebnisse.

Subjektive /selbst wahrgenommene Gesundheit: Insgesamt 57,9 \% der im Jahr 2019 befragten Eltern und pflegenden Angehörigen bewerteten ihre Gesundheit mit gut $(46,6 \%)$ oder sehr gut $(11,3 \%)$, weitere 29,9 Prozent mit zufriedenstellend. Insgesamt zwölf Prozent hielten sie für mangelhaft $(8,4 \%)$ oder schlecht (3,6\%). Die überwiegend positive Gesamtbewertung ist dem gegenüberzustellen, dass im Detail viele Belastungen, Gesundheitsstörungen, Beschwerden und Erkrankungen genannt wurden. Andere repräsentative Erhebungen plausibilisieren das Ergebnis. Die Verteilung der in der GEDA-Befragung mit der Skala des international bewährten „Minimum European Module (MEHM)“ erhobene subjektive Gesundheit sah im Jahr 2014/15 wie folgt aus: $14,8 \%$ sehr gut, $53,4 \%$ gut, $26,3 \%$ mittelmäßig, 4,8 \% schlecht und 0,7\% sehr schlecht. ${ }^{4}$

Gesundheitsstörungen: Im Durchschnitt nannten die Befragten sechs

$274 \%$ der Widersprüche erfolgreich; $2018=70 \%$

3 Auf die Frage, inwieweit die Validität von „objektiven“ ärztlichen Diagnosen durch eine Fülle von subjektiven Faktoren in der Person des Arztes/der Ärztin oder der in einer Institution gegebenen diagnostischen Standards oder Leitlinien beeinflusst wird, soll an dieser Stelle nicht eingegangen werden.

4 https://www.rki.de/DE/Content/Gesundheitsmonitoring/ Gesundheitsberichterstattung/GBEDownloadsJ/FactSheets/ JoHM_02_2018_Subjektive_Gesundheit_Erwachsene.pdf?_blob=publicationFile 
Tabelle 2: Gesundheitsstörungen der befragten Eltern und pflegenden Angehörigen

\begin{tabular}{|l|c|}
\hline Leiden Sie schon länger an einer der folgenden Gesundheitsstörungen? & Anteil ja in \% \\
\hline häufig Rücken-, Schulter- oder Nackenbeschwerden & 47,8 \\
\hline ständig müde & 40,8 \\
\hline stark erschöpft oder das Gefühl, ausgebrannt zu sein & 40,6 \\
\hline häufig Probleme sich zu entspannen & 38,4 \\
\hline häufig lustlos & 38,4 \\
\hline Unter- oder Übergewicht & 36,0 \\
\hline häufig Schlafstörungen & 34,3 \\
\hline häufig gereizt & 30,6 \\
\hline häufig niedergeschlagen & 30,5 \\
\hline häufig Stimmungsschwankungen & 30,4 \\
\hline häufig sonstige Schmerzen & 30,4 \\
\hline körperliche Leistungsfähigkeit häufig eingeschränkt & 28,1 \\
\hline häufig Kopfschmerzen & 25,2 \\
\hline häufig vergesslich & 24,1 \\
\hline häufig Konzentrationsstörungen & 24,0 \\
\hline häufig Magen-Darm-Probleme & 23,6 \\
\hline häufig unruhig oder häufig Angstgefühle & 4 \\
\hline
\end{tabular}

Quelle: Sommer et al. (2021), S. 32

Gesundheitsstörungen. Nur jede/jeder Neunte nannte gar keine. Die häufigsten Störungen waren Rücken-, Schulter- oder Nackenbeschwerden oder Müdigkeit (vgl. Tabelle 2).

Beeinträchtigungen: Durch einen Mangel an Lebensfreude, wenig Interesse oder Freude an den eigenen Tätigkeiten, eine schlechte Meinung von sich oder das Gefühl versagt oder die Familie enttäuscht zu haben, fühlte sich rund ein Viertel bis ein Fünftel der Befragten an mehr als der Hälfte der Tage oder beinahe jeden Tag beeinträchtigt.

Erkrankungen: Die Befragten Eltern und pflegenden Angehörigen nannten darüber hinaus durchschnittlich ein bis zwei $(\varnothing 1,3)$ Erkrankungen, aufgrund derer sie schon seit längerer Zeit in medizinischer Behandlung waren. Nur jede/ jeder Dritte gab hier keine Erkrankung an. Am häufigsten waren Verschleißerscheinungen an der Wirbelsäule und den Gelenken, Herz-Kreislauf-Erkrankung sowie Hauterkrankungen und Allergien.

Belastungen: Für die Vorsorge- oder Rehabilitationsleistungen in Mütter-/ Väter/-Kind-Einrichtungen ist das Wechselspiel zwischen den Gesundheitsstörungen, Beeinträchtigungen und Erkrankungen mit elternspezifischen, familiären und psychosozialen Belastungen oder weiteren Risikofaktoren entscheidend. Knapp jede oder jeder Vierte $(24,4 \%)$ war nach eigenen Angaben frei von allen Belastungen aus diesen Bereichen. Durchschnittlich wurden drei bis vier solcher Belastungen genannt (Ø 3,5 Nennungen). Psychosoziale Belastungen (z. B. Finanzielle Sorgen, Zeitdruck, soziale Isolation oder Arbeitslosigkeit) sind häufiger $(\varnothing 1,3)$. Seltener werden elternspezifische (z. B. schwere Vereinbarkeit von Familie und Beruf, Erziehungsschwierigkeiten, Trennungen, oder erhöhter Betreuungsaufwand durch chronische Krankheiten oder Behinderungen der Kinder), familiäre Belastungen (z. B. mangelnde Anerkennung, Tod von Familienangehörigen oder Pflege von Angehörigen) oder weiteren Risikofaktoren (z. B. Bewegungsmangel, ungesunde Ernährung, Rauchen oder Probleme mit Alkoholkonsum) angegeben (je Ø 0,6 bis 0,8$)$.

Anstieg: Die Erhebung war auch so konzipiert, einen Vergleich mit einer Vorgängerstudie aus dem Jahr 2007 zu ermöglichen. ${ }^{5}$ Deutlich ist ein Anstieg vieler der zuvor genannten Indikatoren. Viele der Gesundheitsstörungen wurden rund doppelt so häufig oder noch häufiger angegeben wie im Jahr 2007. Im Jahr 2019 nannten z. B. 36 \% häufige Schlafstörungen, zwölf Jahre zuvor galt dies für 14,3 \% der Frauen und 7,4 \% der Männer. ${ }^{6}$ Externe Quellen plausibilisieren das Ergebnis, z. B. erhöhten sich Schlafstörungen laut Daten des DAK-Gesundheitsreports zwischen 2010 und 2017 um 66 Prozent. $^{7}$ Die gleiche Tendenz zeigen Angaben zu häufigen Kopfschmerzen (im Jahr 2019 28,1%) gegenüber den viel niedrigeren Daten aus dem Jahr 2007 (19,5\% bei Frauen und $8,8 \%$ bei Männern), ${ }^{8}$ die sich wiederum mit den Ergebnissen einer 2019/20 bundesweit umgesetzten Befragungsstudie des Robert Koch Instituts (RKI) ${ }^{9}$ vergleichen lassen.

Einzelfallbezogene Definition des Bedarfs: Die Begutachtungsanleitung der $G_{K V}{ }^{10}$ zählt analog zu den oben aufgeführten Indikatoren vielfältige Gesundheitsstörung oder Erkrankung, Umwelt- und personenbezogenen Faktoren (bzw. psychosoziale Belastungen und belastende Umstände) sowie mütter- $/$ väterspezifische Kontextfaktoren (bzw. elternspezifische und familiäre Belastungen) zu den möglichen Indikatoren eines Bedarfs stationärer Vorsorge- bzw. Rehabilitationsleistungen in Mutter-/ Vater/Kind-Einrichtungen. Diese Merkmale indizieren laut der GKV einen tatsächlichen Vorsorgebedarf jedoch noch nicht hinreichend. In der Formulierung heißt es nur, dass diese Merkmale eine Indikation darstellen „können“. Notwendig ist, dass die Ärztin oder der Arzt eine einzelfallbezogene Gesamtbetrachtung und den kausalen Zusammenhang zwischen diesen Merkmalen attestiert in dem Sinn, dass „die im Antragsverfahren erkennbaren Kontextfaktoren (...) im Zusammenhang mit der Erziehungsverantwortung zu einer mütter-/ väterspezifischen Problemkonstellation führen, die das Gesundheitsproblem bedingt, unterhält oder verstärkt und eine Vorsorgeleistung in einer Mütter-/Väterbzw. Mütter-/Väter-Kind-Einrichtung erfordert" (S. 29). Darüber hinaus muss zusätzlich auch die „Notwendigkeit ei-

5 Faßmann et al. (2008)

6 ebd. S. 170

7 Zitiert nach https://www.ndr.de/ratgeber/ gesundheit/Schlaf-Studie-Deutsche-oftmuede-zur-Arbeit,schlafen154.html.

8 Vgl. Faßmann et al. (2008) S. 176.

9 57,5\% der Frauen und 44,4\% der Männer berichten, binnen eines Jahres mindestens einmal von Kopfschmerzen betroffen zu sein. 14,8\% der Frauen und 6,0\% der Männer erfüllen die Kriterien für Migräne (Porst et al. 2020: S. 2)

10 Medizinischer Dienst des Spitzenverbandes Bund der Krankenkassen (2018): Vgl. dort S. $27 \mathrm{ff}$. zu den Kriterien der Vorsorge und S. 36 ff. für die Kriterien zur Rehabilitation. 
ner Herausnahme aus dem häuslichen Umfeld“ (S. 27) gegeben sein. ${ }^{11}$

Statistische Definition des Bedarfs: Auf Basis unserer Befragungsdaten war eine einzelfallbezogene Feststellung des Vorsorge- oder Reha-Bedarfs nicht möglich. Über die parallele Befragung von ehemaligen Patient:innen konnte jedoch ein statistischer Indikator für den Bedarf erschlossen werden. Die Patient:innen wurden zu Gesundheitsstörungen, Erkrankungen und Belastungen befragt, die sie vor Antritt der Vorsorge- oder Reha-Leistungen in Einrichtungen des MGW hatten - und die damals offensichtlich von einer Ärztin oder einem Arzt als ausreichend angesehen wurden, den Vorsorge- oder Reha-Bedarf zu attestieren. Die Angaben zu Belastungen, Gesundheitsstörungen, Erkrankungen und Beeinträchtigungen wurden zu einem Gesamtindex des Vorsorge- und Reha-Bedarfs aggregiert. Die Verteilung dieses Gesamtindex unter den Patient:innen des MGW lieferte einen Referenzwert. Haben andere Eltern und pflegende Angehörigen einen gleichen oder höheren Index-Wert ihrer Gesundheitsstörungen, Erkrankungen und Belastungen, kann angenommen werden, dass eine Ärztin oder ein Arzt mit hoher Wahrscheinlichkeit den Vorsorgeoder Reha-Bedarf attestiert. (vgl. Näheres zur Methode und Berechnung in Sommer et al. 2021: S. 30, $34 \mathrm{ff}$ ).

Ergebnisse zum Bedarf: Nach dieser Berechnung benötigen insgesamt 18,9\% aller Mütter, Väter und pflegenden Angehörigen aufgrund ihrer gesundheitlichen und Belastungssituation eine Rehabilitations- oder Vorsorgemaßnahme. Der Anteil der Personen mit Bedarf sah differenziert nach Untergruppen so aus:

- 23,9 \% der Frauen und 13,8 \% der Männer,

- $33 \%$ der Mütter und Väter, die Angehörige pflegen und

- 75 \% der Eltern von Kindern mit einer Behinderung.

In Hinblick auf die Väter bestätigt sich einerseits, dass ihr Bedarf an diesen Maßnahmen deutlich niedriger liegt als der von Müttern. Aufgrund der geschlechtsspezifischen Arbeitsteilung ist gut. dies nicht verwunderlich. Andererseits ist ihr Bedarf nicht gleich null. Würde die Nutzung der Maßnahmen dem Bedarf entsprechen, müsste das Verhältnis von Patienten zu Patientinnen im MGW bei rund 1:2 liegen. Der faktische Anteil der Männer liegt im unteren einstelligen Prozentbereich und die Ablehnungsquote der Erstanträge liegt bei Vätern deutlich höher als bei Müttern. Für Väter sind die individuellen, sozialen oder institutionellen Hürden der Inanspruchnahme noch deutlich höher als für Mütter.

\section{Zugang}

Von den durch unsere Befragung identifizierten Müttern, Vätern und pflegenden Angehörigen mit einem nach unseren Kriterien behandlungsbedürftigen gesundheitlichen Zustand überlegten sich nur 21,9\%, eine stationäre Maßnahme zu beantragen. $\mathrm{Zu}$ den Gründen zählt lediglich bei etwas mehr als einem Drittel die Unkenntnis des Angebots. Die vor oder während einer Beantragung von Mutter/Vater-/Kind (MVK)-Maßnahmen sinnvolle Beratung in einer der da-

$51 \%$ der ehemaligen Patient:innen nutzten vor einer Maßnahme eine Beratungsstelle und $92 \%$ von ihnen bewerteten deren Beratungsleistung mit gut/sehr

mals rund 960 existierenden (tendenziell sinkenden Zahl) Beratungsstellen kommt nicht zustande oder scheitert an der Unbekanntheit, dem Fehlen in erreichbarer Nähe, dem Mangel an gezielter Weiterbildung von Mitarbeitenden (die häufig in Teilzeit die Beratung erbringen), ihrer Überlastung oder an langen Wartezeiten (durchschnittlich neun Tage, relativ häufig auch über drei Wochen). $51 \%$ der ehemaligen Patient:innen nutzten vor einer Maßnahme eine Beratungsstelle und $92 \%$ von ihnen bewerteten deren Beratungsleistung mit gut/sehr gut. $\mathrm{Zu}$ den subjektiven Gründen trotz gesundheitlichen Beeinträchtigungen und hoher alltäglichen Belastungen keine MVKMaßnahme zu beantragen gehört z.B. die im Vergleich mit „richtig Kranken“ unterschätzende Bewertung des eigenen Bedarfs, die lange Wartezeit bis zum Beginn einer Maßnahme, ${ }^{12}$ vermutete Nachteile am Arbeitsplatz, die Scheu von pflegenden Angehörigen ihre Pflegebedürftigen während der Inanspruchnahme einer Maßnahme in fremde Hände zu geben ${ }^{13}$ oder den/die Partner(in) oder Kinder allein zu lassen.

\section{Wirkung und Nutzen von MVK}

Je nach Blickwinkel oder aus welchen Datenquellen sie stammen, unterscheiden sich die Angaben zur Wirkung bzw. zum Nutzen von MVK-Maßnahmen.

Dabei handelt es sich um die Ergebnisse mehrerer wirkungsbezogenen Fragen an ehemalige Patient:innen von Rehabilitations- und Vorsorgemaßnahmen für Mütter und Väter, Einschätzungen von Ärzten/Ärztinnen, die Atteste für die Antragstellerinnen und Antragsteller solcher Maßnahmen erstellen und schließlich um Ergebnisse der auch für die MVK-Rehabilitation gesetzlich durch $\$ 135$ a SGB V vorgeschriebenen externen vergleichenden Qualitätssicherung QS Reha®.

\subsection{Wirkung in der Wahrnehmung von ehemaligen Patientinnen/Patienten}

Wie die ehemaligen Patient:innen die Wirkung und den Nutzen ihrer vor einigen Monaten beendeten Rehabilitations- und Vorsorgemaßnahme beurteilen, konnten sie durch Antworten auf mehrere Fragen zum Ausdruck bringen.

Auf die Frage wie sie ihren allgemeinen Zustand vor, nach Beendigung und zum aktuellen Zeitpunkt, also rund ein halbes Jahr nach Beendigung der stationäre Vorsorge- oder Rehabilitationsmaßnahme beschreiben würden, antworteten 71,4 $\%, 5,6 \%$ und $18,4 \%$ mit mangelhaft

11 Eine ambulante Erbringung komplexer Vorsorgeleistungen ist nach § 24 SGB V nicht vorgesehen.

12 Laut den Mitarbeiter:innen der Beratungsstellen beträgt diese Wartezeit für Mütter 5,9 Monate und für pflegende Angehörige 7,4 Monate.

13 Dass dies auch auf der Unkenntnis der wenigen, aber nutzbaren Angebote von MVK-Maßnahmen mit Pflegebedürftigen oder der vorhandenen aber ebenfalls unübersichtlichen oder unbekannten Möglichkeiten von Ersatzbetreuung während der eigenen Maßnahme beruhen kann, sei der Vollständigkeit halber erwähnt. 
oder schlecht. Und für dieselben Zeitpunkte gaben $94,8 \%, 27,6 \%$ und 51,9 $\%$ an, sich durch die familiäre, elterliche, berufliche oder darüber hinausgehende Situation eher oder sehr belastet gefühlt zu haben bzw. zu fühlen. ${ }^{14}$ Der großen unmittelbaren Wirkung der Maßnahmen folgte also ein mehr oder weniger großer Rebound. Dies verdeutlicht, wie wichtig die Bekanntheit und der Nutzen von Nachsorgemöglichkeiten für die Nachhaltigkeit der Wirkungen ist.

Da die Mehrheit der Nutzerinnen und Nutzer der MVK-Maßnahmen an Erschöpfung litt, zeigt allein der Anteil von $91 \%$ der befragten Patient:innen, die angaben, in der Maßnahme wieder Kraft getankt zu haben, dass die Maßnahmen wirksam waren. Eine deutliche Mehrheit der befragten Patient:innen gibt darüber hinaus zahlreiche Lerneffekte an: Fünf von sechs geben an, sie hätten gelernt, wie sich Stress und Überlastungen auf ihren Körper auswirken, im Alltag besser auf ihre Gesundheit acht zu geben, und die Behandlung habe ihnen geholfen, besser mit ihren Problemen umzugehen. Weniger stark sind die Lerneffekte hinsichtlich der Aufgabe, sich vor dem Hintergrund körperlicher Beeinträchtigungen mit den sozialen Ursachen der Probleme auseinanderzusetzen, z. B. im Beruf oder in der Familie. Mit den körperlichen Beeinträchtigungen im Alltag umzugehen, sich mit den Belastungen als Mutter/Vater/Pflegende oder im Beruf auseinanderzusetzen, hat jeweils nur rund die Hälfte der Befragten bestätigt. Auch wenn sich nur im Einzelfall bewerten lässt, welchen Stellenwert diese Ursachen für die Gesundheit haben, könnte das ein Hinweis darauf sein, wo die Wirkung oder die Nachhaltigkeit der Maßnahmen optimiert werden kann.

Insgesamt ist gut die Hälfte der Befragten mit der erhaltenen Unterstützung und der Art der Behandlung voll und ganz zufrieden, zuzüglich eines guten Drittels, die eher zufrieden sind (rund $10 \%$ unzufrieden, $3 \%$ ohne Angaben).

Wirkungen entfalten die Maßnahmen nicht (oder wahrscheinlich nicht überwiegend) durch „die Auszeit vom Alltag“, sondern durch die verschiedenen Angebote, welche die Patient:innen im Rahmen der Maßnahme in Anspruch nahmen. Je mehr oder je stärker sie diese Angebote in der Maßnahme nutzten, umso stärker sind verschiedene Wirkungen. Die Intensität der Nutzung steht in signifikantem Zusammenhang mit dem, was die Patient:innen dort nach eigenen Angaben lernten, wie sich die Gesundheit kurzfristig und auch langfristig entwickelte und auch, wie das Belastungsgefühl kurzfristig und auch langfristig abnahm.

Auch für die verschiedenen Angebote der Nachsorge lassen sich entsprechend Effekte aufzeigen. Die langfristige Entwicklung der Gesundheit und die Abnahme des Belastungsgefühls stehen in signifikantem Zusammenhang damit, wie Angebote der Nachsorge genutzt wurden. Zusammenhänge lassen sich auch zwischen der individuellen Nutzung bestimmter Nachsorgeangebote und den erwünschten Entwicklungen aufzeigen, insbesondere zu den langfristigen Gesundheitseffekten, die z. B. positiver waren, wenn die Patient:innen eine psychotherapeutische Gesprächstherapie begannen oder fortsetzten, die in der Maßnahme getroffenen Vereinbarungen persönlicher Zielen und nächster Schritten nutzten oder einen in der Maßnahme geschriebenen Brief an sich selbst beachteten, der ihnen nach einiger Zeit von der Klinik zugeschickt wurde oder wenn sie eine Selbsthilfegruppe besuchten, oder sie die Betreuung ihrer Kinder nun anders organisierten. Angesichts des statistisch bestätigten positiven Effekts von Nachsorge ist die von den ehemaligen Patient:innen berichtete und damit auch handlungsbestimmend erinnerte eher geringe Rolle der Nachsorgeempfehlungen in der gesamten Behandlung problematisch.

\subsection{Wirkungen aus Sicht von Ärztinnen und Ärzten}

Von den vor allem wegen ihren Erfahrungen mit einem neuen Musterformular zur Begutachtung der Rehabilitationsbedürftigkeit befragten niedergelassenen Ärztinnen und Ärzten hielten rund $75 \%$ langfristige Therapieerfolge durch MVKRehabilitations- und Vorsorgemaßnahmen für eher oder voll zutreffend. Die Beurteilung unterscheidet sich zwischen den Zielgruppen erheblich: Während Ärzte und Ärztinnen auch einen langfristigen Therapieerfolg für Mütter für zutreffend hielten, sahen dies bei Vätern nur noch $42 \%$ und bei pflegenden An- gehörigen noch $30 \%$ von ihnen so. Und der Anteil der Befragten, die angaben, dies nicht beurteilen zu können, bewegte sich bei diesen beiden Gruppen zwischen $37 \%$ und $54 \%$. Offensichtlich liegt dies auch an der immer noch geringen Anzahl von Vätern und pflegenden Angehörigen, die eine MVK beantragten, in Anspruch genommen und ihrer Ärztin oder ihrem 
der Bewertung von sieben Einzelwerten zur Gesundheit und zehn Einzelfragen zu mütter-/väterspezifischen Kontextfaktoren zu Beginn und sechs Wochen nach Beendigung der Maßnahme. Der Vergleich der Mittelwerte der dabei genutzten Antwortskala beider Erhebungen liefert sogenannte Cohens d-Werte zur Effektstärke. Statistisch gilt der Effekt bei Werten von 0,2-0,5 als klein, von 0,5-0,8 als mittel und groß bei Werten über 0,8 .

Die Effektstärke der Mutter/VaterRehabilitation und -Vorsorge je ehemaliger Patient:innen liegt bei 11 von 17 Indikatoren der Ergebnisqualität mehr oder weniger weit unter 0,5 und ist damit im statistischen Sinn klein oder nicht messbar. Vier Indikatoren haben eine mittlere und zwei eine große Effektstärke. Unterscheidet man Qualitätsunterdimensionen, die sich eher auf Gesundheitsprobleme beziehen oder eher etwas mit mütter-/väterspezifischen Kontextfaktoren zu tun haben, gibt es große Effekte nur bei zwei der sieben Gesundheitsproblem-Indikatoren. Bei den zehn Kontextfaktoren-Indikatoren nehmen die befragten ehemaligen Patient:innen keinen großen Effekt wahr und die meisten auch nur kleine Effektstärken. An den geringen Wirkungsstärken hat sich seit 2015 nichts verändert.

\section{Wirkungen durch Angebot und Nutzung von Nachsorge}

MVK-Maßnahmen können in der Regel frühestens vier Jahre nach einer Inanspruchnahme wiederholt werden und Patient:innen kehren nach Beendigung einer Maßnahme sofort wieder in jene wahrscheinlich kaum veränderten familiären und sonstigen sozialen Verhältnisse zurück, die mit zu ihrer Rehabilitations- oder Vorsorgebedürftigkeit beigetragen haben. Daher sind nachhaltigkeitssichernde Leistungen während des stationären Aufenthalts wie z.B. Informationen über und die Vorbereitung auf Nachsorgemöglichkeiten und natürlich bei Bedarf die Inanspruchnahme solcher Angebote unbedingt notwendig. Nachsorge stellt auch einen integralen Bestandteil von Wirtschaftlichkeit dar. Wirtschaftlich ist „nicht die billigste zweier notwendigen Leistungen, sondern diejenige mit der besten Kosten-Nutzenrelation (...) der Nutzen bestimmt sich nach dem Be- handlungsziel unter Einbeziehung der Nachhaltigkeit des Heilerfolgs, der Lebensqualität des Versicherten sowie der in anderen Leistungsbereichen und Sozialversicherungszweigenvermiedenen bzw. bewirkten Kosten." (Becker und Kingreen 2018, S. 132 zum \12 SGB V)

Bei der Bewertung des Angebots von auf die Nachsorge bezogenen Leistungen während der Rehabilitation oder Vorsorge, ist zu beachten, dass sich fast alle der von uns befragten Patient:innen zu Beginn ihres Aufenthalts in äußerst schlechter Verfassung befanden. $95 \%$ fühlten sich durch familiäre Kontextfaktoren eher oder sehr belastet und $51 \%$ bewerteten ihre gesundheitliche Verfassung als mangelhaft.

Trotzdem antworteten auf die Frage, welche von neun möglichen Nachsorgeangeboten (z.B. Vereinbarung von Zielen, Kooperation mit Beratungsstellen, Vermittlung von „nach der Kur“Angeboten) sie nie, im Einzelfall oder systematisch anbieten, zwischen $2 \%$ und $13 \%$ der Einrichtungen dies erfolge bei fünf Angeboten „nie“. Der Anteil der Einrichtungen, die jedes der neun Angebote „im Einzelfall“ anbieten bewegt sich zwischen $24 \%$ und $89 \%$. Und „systematisch“, d.h. für die große Mehrheit der Patient:innen bieten diese Angebote zwischen $2 \%$ und $76 \%$ der Einrichtungen an.

Angesichts des geschilderten Belastungs- und Gesundheitszustands der Neuankömmlinge ist es zwar positiv zu bewerten, dass mehr als $50 \%$ bis maximal $75 \%$ der Einrichtungen sagen, vier dieser Angebote systematisch anzubieten. Trotzdem erhalten die Patient:innen in einem Viertel bis fast der Hälfte der Einrichtungen selbst diese vier Angebote lediglich im Einzelfall und damit möglicherweise nicht. Noch deutlicher gilt dies für die fünf Angebote, die nur in deutlich weniger als 50 Prozent der Einrichtungen systematisch erbracht werden.

$\mathrm{Zu}$ welchen quantitativen und qualitativen Lücken ein Einzelfallangebot von Hinweisen auf die Relevanz und die Möglichkeiten von nachstationärer Nachsorge führen kann, zeigt eine entsprechende Befragung der ehemaligen Patient:innen: Nur bei fünf von $21 \mathrm{er}-$ fragten Nachsorgeleistungen sagen mehr als $50 \%$ der Befragten, sie wären ihnen empfohlen worden bzw. sie hätten sie erhalten. Zwei der Empfehlungen beziehen sich auf das Gesundheitsverhalten und zwei weitere sind Instrumente zur Selbstverpflichtung der Patient:innen. Zwischen 26 und $50 \%$ der Patient:innen gaben an, dass ihnen drei weitere nachsorgerelevante Leistungen während der Kur empfohlen wurden. Darunter sind sowohl eine weitere auf das Gesundheitsverhalten bezogene Leistung als auch eine therapeutische Empfehlung. 13 weitere Nachsorgeleistungen wurden lediglich noch zwischen 17 und 0,3\% aller Patient:innen empfohlen bzw. für

\section{Nachhaltigkeitssichernde eistungen sind während des stationären Aufenthalts dingt notwendig.}

sie oder mit ihnen organisiert. Neben den zwei gesundheitsbezogenen Empfehlungen, eine Selbsthilfegruppe zu besuchen und sich z. B. in der Apotheke nicht verschreibungspflichtige Arzneimittel oder Nahrungsergänzungsmittel zu besorgen, handelt es sich der Mehrheit der Leistungen um Empfehlungen oder Hinweise auf institutionelle und auf familiäre Kontextfaktoren bezogene Beratungs- und Unterstützungsressourcen. Etwas mehr als $50 \%$ der Patient:innen erhielten für 16 der 21 nachsorgebezogenen und -relevanten Leistungen in ihrer Erinnerung keine Empfehlung. Für über $80 \%$ traf dies für 13 von 21 dieser Art von Leistungen zu.

Dass möglicherweise der Gesundheitszustand vor und zu Beginn der stationären Behandlung nicht die von uns für notwendig gehaltene Rolle gespielt hat, zeigt sich darin, dass es keinen statistisch signifikanten Zusammenhang von selbst wahrgenommenem Gesundheitszustand und der Anzahl der vorstationär behandelten Erkrankungen mit der Häufigkeit gesundheitsbezogener Nachsorgeempfehlungen gab. Signifikant wird dieser Zusammenhang bei Fokussierung auf die Anzahl von vorstationären Gesundheitsstörungen. Und auch der Zusammenhang von selbst wahrgenommener starker Belastung durch soziale und familiäre Umstände mit spezifischen Nachsorgeempfehlungen ist nicht signifikant. Von den sehr oder eher belasteten Personen haben $18 \%$ gar keine und weitere $27 \%$ 
nur eine belastungsbezogene Nachsorgeempfehlung erhalten.

Auf die Frage, wie viele der gesundheits- und belastungsbezogenen Nachsorgeempfehlungen sie zum Befragungszeitpunkt, also mehrere Monate nach Beendigung der Maßnahme nutzen oder vorhaben sie zu nutzen, bewegt sich der Anteil der ehemaligen Patient:innen zwischen maximal $47 \%$, die Sport machen und minimal $1 \%$, die eine Haushaltshilfe bei der Krankenkasse beantragt haben. Einige der auf das Gesundheitsverhalten bezogenen Nachsorgeangebote (Essen, Entspannung) werden von rund einem Drittel aller Befragten genutzt. Zehn der 17 Angebote werden von weniger als $10 \%$ aller befragten ehemaligen Patient:innen genutzt. Maximal $19 \%$ von ihnen haben vor, noch Sport- und Entspannungs-Angebote zu nutzen.

Von den Befragten, die während ihrer Maßnahme Nachsorgeempfehlungen erhielten, nutzen durchweg mehr diese Angebote. Der Anteil der Nutzenden reicht von maximal $64 \%$ bei gesunder Ernährung bis minimal $12 \%$, wenn es um die Nutzung eines Nachsorgeangebots der Klinik geht.

Zu den Gründen für die Nichtnutzung und damit des Verlustes von nachhaltigem Nutzen zählen neben den fehlenden oder nicht als solche bei den Patient:innen „angekommenen“ Empfehlungen fehlende Anbieter in der Wohnumgebung, zu teure Anbieter von Nachsorgeleistungen, der Alltagstrott, fehlende freie Plätze in regionalen Einrichtungen oder die fehlende Kinderbetreuung während des Besuchs von Kursen.

\section{Versorgungspolitische Schlussfolgerungen}

Die zahlreichen sichtbar gewordenen Diskrepanzen zwischen gesundheits- und belastungsbezogenen Bedarfen von Müttern, Vätern und pflegenden Angehörigen und einem quantitativ wie qualitativ adäquaten und unaufwändig zugänglichen Angebot von stationären Vorsorge- und Rehabilitationsangeboten und nachstationärer Nachsorge, erfordern möglichst gleichzeitige komplexere Interventionen. Dazu gehören u.a.

- eine ausreichende, d.h. gegenüber dem Status quo höhere und leicht zugängliche Anzahl von Beratungsstellen, stationären Versorgungsplätzen und Nachsorgeangeboten mit bedarfsgerecht qualifiziertem Personal,

- der gesetzliche Anspruch auf ein nachhaltiges Leistungsangebot, das zusätzlich zur stationären Leistung vor- und nachstationäre Beratungsan- gebote und zur Bewältigung der nach der Inanspruchnahme der Leistungen möglicherweise erneut auftretenden gesundheitlichen Beeinträchtigungen und Belastungen durch familiäre Kontextfaktoren die systematische Beratung über existierende Nachsorgeangebote und deren barrierefreie Nutzbarkeit umfasst,

- die Bildung oder Förderung bedarfsangemessener multiprofessioneller Teams in den Leistungseinrichtungen,

- die Spezifizierung des Leistungsangebots für die neuen Zielgruppen Väter und pflegende Angehörige und Verbesserung der Transparenz über diese Angebote,

- eine auf einer Basisvergütung erfolgende Differenzierung der Honorierung von Leistungen nach unterschiedlichen Bedarfen und Aufwendungen,

- die Erprobung einer Honorierung, die sich an der nachstationären Nachhaltigkeit von Behandlungszielen orientiert und

- die der bestmöglichen Versorgung dienende Klärung der historisch gewachsenen Doppel-Zuständigkeiten für Einrichtungen der Bundesministerien für Gesundheit sowie Familie, Senioren, Frauen und Jugend bzw. die Förderung ihrer Zusammenarbeit.

\section{Literatur}

Bastin S., K. Unzicker (2021): Verlieren die Eltern ihr Vertrauen? Belastungen und Politikvertrauen während der Corona-Pandemie nach Familien-Kontext. Bremen (unveröffentlichtes Manuskript)

Becker U., Kingreen Th. (Hrsg.) (2018): SGB V Gesetzliche Krankenversicherung. Kommentar. (6. Neu bearbeitete Auflage). München

Braun B. (2021) Anspruch und Wirklichkeit von Qualitätssicherung am Beispiel des QS Reha ${ }^{\circledR}$ Verfahrens für die Rehabilitation und Vorsorge in der GKV. In: Gesundheits- und Sozialpolitik Heft 2 64-71

Bujard M., Driesch E.v.d., K. Ruckdeschel, I. Laß, C. Thönnissen, A. Schumann, N. F. Schneider (2021): Belastungen von Kindern, Jugendlichen und Eltern in der Corona-Pandemie. BiB-Bevölkerungsstudien 2. Wiesbaden

Faßmann, H. et al. (2008): Bedarfs- und Bestandsanalyse von Vorsorge- und Reha-Maßnahmen für Mütter und Väter in Einrichtungen des Deutschen Müttergenesungswerkes
(MGW). Abschlussbericht zu einem Forschungsprojekt des BMFSFJ

GKV / MDS (2012): Umsetzungsempfehlungen des GKV-Spitzenverbandes, der Verbände der Krankenkassen auf Bundesebene und des MDS im Zusammenhang mit Anträgen auf Leistungen zur medizinischen Vorsorge und Rehabilitation für Mütter und Väter nach $\S \S 24,41$ SGB V

Hiekel N., Kühn M. (2021): Mental health before and during the COVID-19 pandemic: the role of partnership and parenthood status in growing disparities between types of families MPIDR Working Paper WP-2021-013. Rostock

MDS - Medizinischer Dienst des Spitzenverbandes Bund der Krankenkassen (2018): Begutachtungsanleitung Richtlinie des GKVSpitzenverbandes nach $\S 282$ SGB V. Vorsorge und Rehabilitation

MGW (2020): Datenreport 2020. URL: https:// www.muettergenesungswerk.de/fileadmin/ user_upload/MGW_Datenreport_2020.pdf [Stand 25.02.2021]
OECD (2021): Caregiving in crisis: Gender inequality in paid and unpaid work during COVID-19

Robert Koch-Institut (Hrsg.) (2020): Gesund heitliche Lage der Frauen in Deutschland. Gesundheitsberichterstattung des Bundes. Gemeinsam getragen von RKI und Destatis. Berlin

Sommer J., Braun B., S. Meyer (2021): Endbericht der „Studie zur Untersuchung der Bedarfe von Müttern/Vätern und pflegenden Angehörigen in Vorsorge- und Reha-Maßnahmen in Einrichtungen des Müttergenesungswerkes“ im Auftrag des Bundesministeriums für Familie, Senioren, Frauen und Jugend (erhältlich unter: https://www.forum-gesundheitspolitik de/artikel/artikel.pl?artikel=2706 - Zugriff am 13.12.2021))

Verordnung medizinischer Vorsorge für Mütte und Väter (Muster 64) gemäß § 24 SGB V 